\title{
Wer, wenn nicht wir?
}

\section{Warum Non-Profit-Organisationen die Möglichkeiten von Social Media nutzen sollten}

\author{
MANUELA RETTIG \\ Manuela Rettig, M.A. Sozialma- \\ nagement, Politikwissenschaftlerin \\ M.A., ist Geschäftsführerin von pro \\ familia Baden-Württemberg. Bis \\ 2006 war sie Mitglied der Online- \\ Redaktion von www.profamilia.de \\ und leitete das Projekt "pro familia \\ goes social media" (Konzept und \\ Gestaltung eines Facebook-Auftritts \\ der pro familia Sexualpädagogik in \\ Baden-Württemberg). \\ Iv.baden-wuerttemberg@profamilia. \\ de
}

\author{
Für viele Menschen gehören sie inzwischen \\ zum selbstverständlichen Alltag. Social-Media- \\ Anwendungen wie Facebook und Twitter bieten \\ auch Organisationen und Einrichtungen der \\ Sozialen Arbeit vielfältige Möglichkeiten von der \\ Öffentlichkeitsarbeit bis zur Personalgewinnung.
}

Social Media Anwendungen bringen weltweit Milliarden Menschen zusammen. Sie eröffnen schnelle, persönliche und nicht-hierarchische Kontakt- und Kommunikationsmöglichkeiten und bieten neue Kanäle für alle Arten von Waren und Dienstleistungen. Viele Non-Profit-Organisationen in Deutschland allerdings setzen noch auf die gute alte Webseite und E-Mail und vernachlässigen die vielfältigen Möglichkeiten, die Social Media mit Blogs, Kommunikationsplattformen, Verkaufsportalen und Kurznachrichtendiensten bieten.

Das Social Web hat sich zum Leitmedium entwickelt, über das sich Menschen austauschen und koordinieren (Ebersbach, Glaser und Heigl 2011, S. 274), sich darstellen und informieren. In der Folge verwischen sich die Grenzen zwischen Nutzern, Konsumenten und Produzenten von Inhalten, Programmen, Produkten und Dienstleistungen. Damit kommen Themen wie Datensammlung und Datenschutz, Markenschutz und Urheberrechte erneut auf die Agenda von politischen und sozialen Akteuren und bringen neue Dynamiken hervor.

Neue Konflikte zwischen den Wirtschaften und Regierungen einzelner Staaten um die Deutungs- und Verwendungshoheit dieser Rechte entstehen. Wem gehören die Daten? Den Nutzern als Produzenten oder denjenigen, die Daten sammeln, aufbereiten, in Datenbanken organisieren und verwerten? Denn wenn nahezu alle Daten dieser Welt auf amerikanischen Servern gespei- chert werden und den Verwertungs- und Zugriffsrechten der USA unterliegen, geht es um Persönlichkeitsrechte einzelner Bürger, aber ebenso um die Autonomie- und Schutzrechte von Bürgern anderer Staaten. Es geht hier aber auch um Wettbewerbsvorteile, die sich aus dem technologischen Vorsprung der Nutzung, Speicherung und Sortierung dieser Daten ergeben. Der Wettlauf zwischen Google, Facebook und anderen hat begonnen. Fragen wie Urheberrechte, Datenschutz einzelner Personen, Recht auf informationelle Selbstbestimmung, Recht auf das eigene Bild wirken sich störend auf das Geschäftsmodell der Sammlung, Aufbereitung und Zuordnung sowie Weitergabe von Daten aus.

Für die verantwortlichen Akteure vieler Non-Profit-Organisationen in Deutschland sind diese Fragen ungelöst. Anstatt zur Lösung beizutragen, blenden sie in der Folge den Einsatz von Social Media Anwendungen wie beispielsweise Facebook aus. Hier sind aber neue sozialpolitische Aufgaben entstanden. Fragen nach Datenautonomie, demokratischen Rechten, Verletzung von Persönlichkeitsrechten müssen gestellt werden und könnten zusammen mit den Nutzern gelöst werden. Die Lösungen sollten als Best Practice zur Verfügung gestellt werden. Dazu ist es aber mindestens erforderlich, mit den Nutzern über Plattformen wie Facebook in Kontakt zu kommen.

Das Social Web verschafft den Menschen ungehinderten Zugang zu Kommunikation, Information und Selbstor- 
ganisation. Es bietet der digitalen Elite die offenen Programmierschnittstellen und offene Software, die Foren des Austauschs, die sie für die Weiterentwicklung eines demokratischen Social Webs benötigen. Es bietet dem Nutzer und der Nutzerin einen offenen, weitgehend kostenlosen und den Bedürfnissen jeweils entsprechenden Zugang und die passende Plattform, um sich auszutauschen, Neues zu schaffen, sich darzustellen und mit anderen zusammenzuschließen. Soziale Ungleichheit besteht nur mehr in der Unmöglichkeit des Zugangs zum Social Web, wenn der Zugang einmal vorhanden ist, stehen prinzipiell sämtliche Möglichkeiten der Nutzung offen. Das ist eine zutiefst demokratische Angelegenheit und die Nutzer wehren sich deshalb auch gegen Einschränkungen ihrer Zugriffsrechte auf Inhalte, Informationen, Musik, Filme und mehr.

Diese neue, nicht-kommerzielle Sphäre müsste Non-Profit-Organisationen eigentlich gefallen. Wäre da nicht die in der Geschichte der Menschheit unabänderliche Tatsache, dass alles seinen Preis hat. E-Mail war gestern, und irgendwie müssen diese neuen Dienste auch bezahlt werden. Der Preis für die Nutzung von Social Media ist der Handel mit den und die kommerzielle Verwertung der Nutzerdaten. Das dreiseitige Geschäftsmodell besteht:

- zwischen den Nutzern, die leicht bedienbare Anwendungen, leicht zugängliche Informationen und einfache Kommunikationsmöglichkeiten bekommen, die den Anforderungen einer hochmobilen und individualisierten Gesellschaft entsprechen und der Überwindung von Raum und Zeit dienen
- den Unternehmen, die den Nutzern ihre Anwendungen und Plattformen kostenlos bereitstellen und deren $\mathrm{Da}$ ten sammeln, aufbereiten und verkaufen oder Werbeplattformen anbieten

- und den Unternehmern, die die Daten der Nutzer kaufen und auswerten, um zielgruppenspezifische Werbung, Produktentwicklung und ihre Kunden besser zu kennen.

Im Ergebnis entsteht zwischen werbenden und datenverarbeitenden Unternehmen und den Nutzern ein Beziehungskreislauf, bei dem wechselseitig immer mehr Daten und Informationen schen und einen sozialpolitischen Auftrag gegeben haben, dürfen sich nicht durch Nicht-Beachtung und Nicht-Nutzung aus dem Social Web heraushalten. Gemäß ihrem Auftrag müssen sie die Menschen erreichen und abholen. Wenn ein Teil des Identitätsmanagements, der Herstellung von Beziehungen und der Informationsbeschaffung in einer postmodernen Gesellschaft notwendigerweise über Social Media hergestellt wird, darf dies nicht ignoriert werden.

Non-Profit-Organisationen sollen Social Media begreifen, sie sollen lernen, mit Social Media umzugehen, sie sollen Ressourcen dafür bereitstellen.

"Social Media muss als neue sozialpolitische

\section{Aufgabe verstanden werden«}

ausgetauscht werden, die Nutzer zu weiterer Preisgabe von Informationen angeregt werden, der aber auch Lernprozesse auf beiden Seiten fördern kann.

Hier beginnt der zweite Kreislauf: ein Prozess des Austauschs und des voneinander Lernens, der die Macht der Nutzer gegenüber den Produzenten der Dienstleistung Social Media und der von Produkten. Die Nutzer befinden sich in ständigem Dialog untereinander, vernetzen sich und stellen eine neue demokratische Kultur dar. Hier kommt die Nutzung von MonitoringInstrumenten ins Spiel. Sie bedeutet nicht nur die Eröffnung von Chancen für Manipulation, sondern ebenso die für Interaktion und Austausch.

Non-Profit-Organisationen in Deutschland, die sich einen pädagogi-
Dann können sie in den gesellschaftlichen Diskurs eingreifen, Best Practice im Umgang mit Nutzerdaten beweisen und die Daten der Nutzer sinnvoll für die Gestaltung des Sozialen einbringen. Und sie sollten online und offline ihre Klienten auf die Gefahren des Verlusts von Privatsphäre und Eigentum an den eigenen Gedanken, über Einschränkungen des Rechts auf Selbstbestimmung und über unsichtbare Manipulationen informieren, indem sie selbst über die Kenntnisse verfügen, mit Social Media umzugehen. Und sie sollten in Kontakt treten mit der neuen digitalen Elite und sich eigene Kompetenzen und Werkzeuge im Umgang mit Social Media erarbeiten.

Und in der Praxis sollen Non-ProfitOrganisationen Social Media als Instrument der Meinungsbildung und Partizi-

\begin{tabular}{|l|l|l|l|l|}
\hline Social Media Marketing & Unternehmen & $\begin{array}{l}\text { Non-Profit- } \\
\text { Organisationen }\end{array}$ & $\begin{array}{l}\text { Anwendbarkeit bei Non- } \\
\text { Profit-Organisationen }\end{array}$ & Bewertung \\
\hline Ziel & Online-Marketing & Non-Profit-Marketing & Ja & Ein Muss \\
\hline Geschäftsmodell & Platzierte Werbung & $\begin{array}{l}\text { Platzierte Informatio- } \\
\text { nen und Werbung }\end{array}$ & Noch nicht & Strategie entwickeln \\
\hline Begrifflichkeit & Umsatzziele & Erfolge & Ja & Erfolgsziele finden \\
\hline Zielgruppen & Kunden & $\begin{array}{l}\text { Mitglieder, Unterstützer, } \\
\text { Spender, Förderer }\end{array}$ & Offen & Offen \\
\hline Soziales Risiko & Nutzer zahlen mit Daten & Nutzer zahlen mit Daten & $\begin{array}{l}\text { Nutzer informieren und } \\
\text { einbinden }\end{array}$ & Möglich \\
\hline
\end{tabular}

Die Unterschiede beim Einsatz von Social Media zwischen Wirtschaftsunternehmen und Non-Profit-Organisationen müssen offen benannt werden. 


\section{Was versteht man eigentlich unter Social Media?}

Social Media (auch Soziale Medien) bezeichnen digitale Medien und Technologien, die es Nutzern ermöglichen, sich untereinander auszutauschen und mediale Inhalte einzeln oder in Gemeinschaft zu gestalten. Soziale Interaktionen und Zusammenarbeit in sozialen Medien gewinnen zunehmend an Bedeutung und wandeln mediale Monologe ("one to many") in sozial-mediale Dialoge ("many to many"). Zudem sollen sie die Demokratisierung von Wissen und Informationen unterstützen und den Benutzer von einem Konsumenten zu einem Produzenten entwickeln. Demnach besteht weniger oder kein Gefälle zwischen Sender und Rezipienten (Sender-Empfänger-Modell). Als Kommunikationsmittel werden dabei Text, Bild, Audio oder Video verwendet. Das gemeinsame Erstellen, Bearbeiten und Verteilen von Inhalt, unterstützt von interaktiven Anwendungen, betont auch der Begriff Web 2.O. Das aufkeimende Interesse an den sozialen Medien ist seit Mitte der 1990er Jahre zu beobachten. Zahlreiche Unternehmen haben damit begonnen, das wirtschaftliche Potenzial dieser Medienform stärker zu nutzen.

www.wikipedia.org

pation von unten inklusive der Öffnung gegenüber Mitgliedern, Unterstützern und Klienten nutzen. Sie können damit neue Lernprozesse anregen, Klientinnen und Klienten in die Entwicklung ihrer Dienstleistungen einbeziehen, sich ebenso über die Wünsche ihrer Klientinnen kundig machen, wie über die Nöte direkt und unvermittelt Bescheid wissen. Sie sollten auch selbstbewusst mit ihren Dienstleistungen werben und Social Media Marketing nutzen, um ihre Dienstleistungen bekannt und zugänglich zu machen. Virales Marketing im Social Web entspricht der Verbreitung von guten Botschaften im realen Leben, auf die Non-Profit-Organisationen angewiesen sind. Sie sollten die Möglichkeiten nutzen, ihre Dienstleistungen $\mathrm{zu}$ verbessern und an die Bedürfnisse der Menschen anzupassen. Mit der Nutzung von Social Media Marketing können Non-Profit-Organisationen

- mehr über ihre Nutzer erfahren, in Austausch treten, von ihnen lernen und Evaluationen starten

- die Nutzer via Werbung für nichtwerbliche Zwecke erreichen

- für ihre Produkte neue Vertriebswege nutzen

- eine offene und vertrauensvolle Kommunikation mit ihren Klienten und Unterstützern führen

- ihre sozialen Themen, ihre Dienstleistungen und politischen Anliegen, sofern sie gehört werden wollen, bei der weiter steigenden Nutzung von Social Media in Social Web verankern. Potenzielle Mitglieder, Förderer, Klienten, Spender, Käufer, Informationssuchende, Menschen in Not, für alle sollten Zugänge im Social
Web geschaffen werden.

- in Krisen schnell Kontakt mit den Nutzern aufnehmen und angemessen reagieren

- die Non-Profit-Organisation als Marke, ihre Mission und Ziele sichtbar werden lassen und eine Plattform des Austauschs bieten.

Über Social Media können serviceorientierte und dialogische Anwendungen bereitgestellt werden. Kunden sollen über Ort, Zeit, Raum und Art der Leistungserstellung informiert werden und Möglichkeiten für Feedback und Beurteilung der Leistung erhalten. Das bedeutet auch, ein Stück Kontrolle abzugeben an die Nutzer und dafür Anregungen, Wissen und gemeinsam getragene Inhalte zu bekommen. Im besten Fall erreichen sie über Social Media weitere Menschen, die für die Organisation, ihre Ziele einstehen, ihr Zeit oder Geld zur Verfügung stellen. Dafür stehen zahlreiche Plattformen zur Auswahl, die entdeckt und ausgewählt werden können.

\section{Literatur}

Bitkom, Bundesverband Informationswirtschaft, Telekommunikation und Neue Medien e. V. Soziale Netzwerke, Zweite erweiterte Befragung. Berlin, www.bitkom.org/files/ documents/BITKOM_Publikation_Soziale_ Netzwerke_zweite_Befragung.pdf. 122011. Ebersbach, Anja, Markus Glaser und Richard Heigl. Social Web. Konstanz 2011, 2. Leistert, Oliver, Theo Röhle (Hg.) Generation Facebook. Über das Leben im Social Web. Bielefeld 2011.

Michelis, Daniel und Thomas (Hg.) Schildhauer. Social Media Handbuch. Baden-Baden 2012.

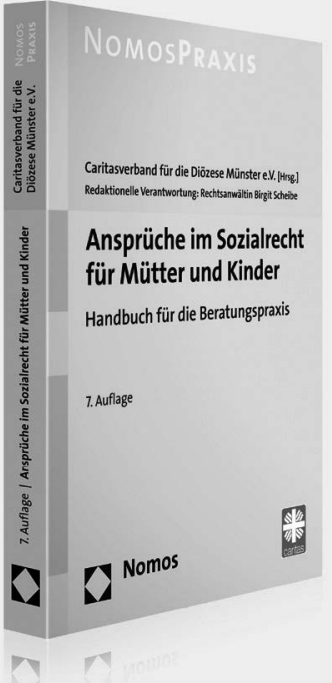

Ansprüche im Sozialrecht für Mütter und Kinder

Handbuch für die Beratungspraxis

Herausgegeben vom

Caritasverband für die Diözese Münster e.V.

Redaktionelle Verantwortung: Rechtsanwältin Birgit Scheibe

7. Auflage 2012, 451 S., brosch., 39,-€, ISBN 978-3-8329-5969-2

Auf aktuellem Stand und natürlich unter Berücksichtigung der Änderungen durch die Hartz-IVReform sowie beim Elterngeld und in der Krankenversicherung bietet die Neuauflage allen Sozialrechtsberatern/-innen systematisch wie praxisorientiert Lösungen zu Fragen der juristischen Beratungspraxis. Dabei werden auch wichtige Fragen der Praxis zum SGB III, MuSchG und Krankenschutz erörtert. Die notwendigen Bezüge zum UVG, Kindergeld und Kinderzuschlag werden hergestellt und Fragestellungen aus der Jugendhilfe und zum BAföG nebst den familienrechtlichen Aspekten aufgegriffen.

www.nomos-shop.de/12910

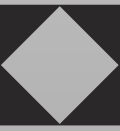

Nomos 